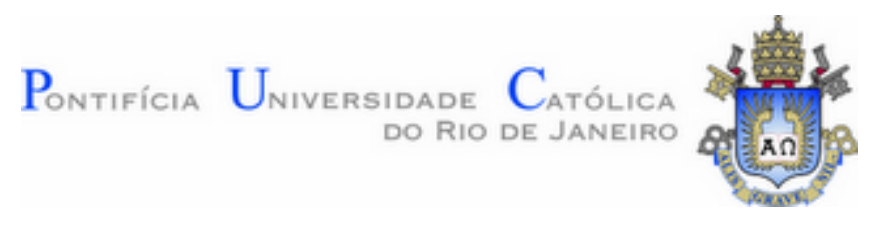

Ernesto Javier Ruano Herreria

Simulação de um ciclo Rankine Orgânico acionado por energia solar

Dissertação de Mestrado

Dissertação apresentada como requisito parcial para obtenção do título de Mestre pelo Programa de PósGraduação em Engenharia Mecânica da PUC-Rio.

Orientador: Prof. Sergio Leal Braga

Rio de Janeiro

Abril de 2012 
Ernesto Javier Ruano Herreria

\title{
Simulação de um ciclo Rankine orgânico acionado por energia solar
}

\begin{abstract}
Dissertação apresentada como requisito parcial para obtenção do título de Mestre pelo Programa de PósGraduação em Engenharia Mecânica da PUC-Rio. Aprovada pela Comissão Examinadora abaixo assinada.
\end{abstract}

Prof. Sergio Leal Braga

Orientador

Departamento de Engenharia Mecânica - PUC-Rio

Prof. José Alberto dos Reis Parise Departamento de Engenharia Mecânica - PUC-Rio

Prof. Carlos Eduardo Leme Nóbrega

Centro Federal de Eduacação Tecnológica Celso Suckow da Fonseca-RJ

Prof. José Eugênio Leal

Coordenador(a) Setorial do Centro Técnico Científico - PUC-Rio 
Todos os direitos reservados. É proibida a reprodução total ou parcial do trabalho sem autorização da universidade, do autor e do orientador.

Ernesto Javier Ruano Herreria

Graduo-se em Engenharia Mecânica pela EPN (Escuela Politécnica Nacional) de Quito, Ecuador em 2009

Ficha Catalográfica

Ruano Herreria, Ernesto Javier

Simulação de um ciclo Rankine orgânico acionado por energia solar / Ernesto Javier Ruano Herreria; orientador: Sergio Leal Braga. - 2012

215 f.: il. (color); $30 \mathrm{~cm}$

Dissertação (mestrado) - Pontifícia Universidade Católica do Rio de Janeiro, Departamento de Engenharia Mecânica, 2012.

Inclui bibliografia.

1. Engenharia mecânica - Teses. 2. Energia solar. 3. Ciclo Rankine orgânico (ORC). 4. Concentradores parabólicos. I. Braga, Sergio Leal. II. Pontifícia Universidade Católica do Rio de Janeiro. Departamento de Engenharia Mecânica. III. Título. 
Dedico este trabalho a minha família pela educação e bons valores recebidos. 


\section{Agradecimentos}

A meus pais, irmãos e toda minha família pelo apoio incondicional, carinho e compreensão.

Ao professor Sergio Leal Braga por sua acertada colaboração no desenvolvimento do presente trabalho.

A meus amigos do Equador e os amigos que fiz durante estes anos no Brasil, pelos bons momentos vividos.

Aos professores e pessoal da PUC-Rio pela valiosa contribução a meu processo educstivo.

Aos órgãos de fomento à pesquisa: CNPq, CAPES e FAPERJ, pelo apoio financiero fornecido, sem o qual este trabalho simplesmente não teria sido possível. 


\section{Resumo}

Ruano Herreria, Ernesto Javier; Braga, Sergio Leal. Simulação de um ciclo Rankine orgânico acionado por energia solar. Rio de Janeiro, 2012. 215p. Dissertação de Mestrado - Departamento de Engenharia Mecânica, Pontifícia Universidade Católica do Rio de Janeiro.

Esta simulação considera um ciclo Rankine que utiliza um fluido de trabalho orgânico, com a particularidade que a fonte de energia de entrada ao sistema será solar. Esta energia renovável que provem do potencial do Sol é aproveitada com a utilização de coletores concentradores lineares parabólicos. Estes dois circuitos: do ciclo Rankine orgânico e do conjunto de coletores interatuam termicamente mediante um trocador de calor chamado de gerador de vapor. Adicionalmente, existe um sistema de armazenamento térmico que permite acumular parte da energia solar coletada para ser utilizada em períodos sem radiação solar ou com níveis baixos da mesma. A primeira parte deste trabalho mostra os aspectos teóricos introdutórios e as considerações para trabalhar com um ciclo Rankine de tipo orgânico, o tipo de coletores escolhido e a utilização de armazenamento térmico. O segundo capítulo mostra o modelo matemático apropriado para simular um sistema de geração de potência de baixa capacidade (50 kW) e os componentes de cada circuito: ciclo (bomba, expansor, condensador, recuperador, gerador de vapor), coletores (cobertura, refletor, absorvedor, etc.) e armazenamento (tanques, etc.). A simulação foi desenvolvida no software EES. O terceiro analisa os parâmetros do modelo, seus possíveis valores físicos, a sensibilidade da sua variação e sua seleção adequada com o objetivo de efetuar uma simulação bastante similar à realidade e as incertezas presentes. No capítulo final se apresentam os resultados em base as condições de desenho consideradas.

\section{Palavras-chave}

Energia solar; ciclo Rankine orgânico (ORC); concentradores parabólicos. 


\section{Abstract}

Ruano Herreria, Ernesto Javier; Braga, Sergio Leal (Advisor). Simulation of an organic Rankine cycle powered by solar energy. Rio de Janeiro, 2012. 215p. MSc. Dissertation - Departamento de Engenharia Mecânica, Pontifícia Universidade Católica do Rio de Janeiro.

This simulation considers a Rankine cycle that works with an organic fluid, but has the particularity of using solar power as the font of input energy. This renewable energy that comes from the sun's potential is taken with the use of parabolic trough collectors. These two circuits: that of the organic Rankine cycle (ORC) and the other of collector's ensemble interact termically in a heat exchanger called as vapor generator. Adicionally there's a thermal storage system that allows accumulating part of the collected solar energy to be used for periods of time when there's no solar radiation or with very low levels of it. The first part of this work shows the introductory theoretical aspects and the considerations to work with an organic Rankine cycle (ORC), the type of chosen collector and the use of heat storage. The second chapter shows the appropriate mathematic model to simulate a system of power generation of low capacity $(50 \mathrm{~kW})$ and the components of each circuit: ORC (pump, expander, condenser, recuperator, vapor generator), collectors (glass cover, reflector mirror, absorber tube, etc.) and thermal storage (storage tanks, etc.). The simulation was developed using EES software. The third chapter analyzes the parameters of the model, specially its values and possible variations to approach the simulation to the reality. In the final chapter, some results are presented based on some considered design conditions.

\section{Keywords}

Solar energy; organic Rankine cycle (ORC); parabolic trough. 


\section{Sumário}

1 INTRODUCÃO

1.1 OBJETIVOS 35

$\begin{array}{ll}\text { 1.2 ENERGIA SOLAR } & 36\end{array}$

1.2.1 Radiação solar $\quad 37$

1.2.2 Tipos de radiação incidente 38

1.2.3 Ângulos solares $\quad 39$

1.3 COLETORES CONCENTRADORES LINEARES PARABÓLICOS 40

1.3.1 Geometria do coletor concentrador linear parabólico 41

1.3.1.1 Definição geral da parábola 41

1.3.1.2 Equação de uma parábola 42

1.3.1.3 Comprimento da parábola 43

1.3.2 Medidas de um coletor concentrador linear parabólico 43

1.3.3 Razão geométrica de concentração solar 44

1.3.4 Reflexão dos raios solares na parábola 45

1.3.5 Ângulo de seguimento do sol para concentradores lineares $\quad 47$ parabólicos

1.3.5.1 Rotação uniaxial ao redor do eixo horizontal oeste - leste com $\quad 47$ ajuste diário

1.3.5.2 Rotação uniaxial ao redor do eixo horizontal oeste - leste com 48 ajuste continuo

1.3.5.3 Rotação uniaxial ao redor do eixo horizontal norte - sul com 48 ajuste continuo

1.3.6 Eficiência óptica do coletor 49

1.3.7 Vantagens dos coletores concentradores lineares parabólicos $\quad 50$

1.3.8 Desvantagens dos coletores concentradores lineares parabólicos $\quad 50$

1.4 CICLO RANKINE ORGÂNICO

1.4.1 Balanço energético do ciclo Rankine orgânico 52

1.4.2 Irreversibilidades no ciclo Rankine 54 
1.4.3 Fluido de trabalho no ciclo Rankine orgânico

1.4.3.1 Motivação para o uso de fluido orgânico num ciclo Rankine 55

1.4.3.2 Características desejadas para a seleção do fluido de trabalho 57 orgânico

1.4.3.2.1 Impacto ambiental $\quad 57$

1.4.3.2.2 Temperatura crítica $\quad 58$

1.4.3.2.3 Pressão crítica $\quad 59$

1.4.3.2.4 Temperatura de degradação $\quad 59$

1.4.3.2.5 Disponibilidade comercial $\quad 59$

1.4.4 Aplicações do ciclo Rankine orgânico 60

$\begin{array}{ll}\text { 1.4.4.1 Recuperação de calor de rejeito } & 60\end{array}$

1.4.4.2 Aproveitamento de energia geotérmica 61

1.4.4.3 Biomassa $\quad 62$

1.4.5 O ciclo Rankine orgânico com energia solar 62

1.5 ARMAZENAMENTO TÉRMICO 65

1.5.1 Tipos de armazenamento térmico 66

1.5.1.1 Armazenamento direto com dois tanques 66

1.5.1.2 Armazenamento indireto com dois tanques 67

1.5.1.3 Armazenamento direto com um tanque simples com efeito 68 termoclina

1.5.1.4 Utilização de sal fundido como fluido térmico nos coletores 68 solares

2 MODELO MATEMÁTICO 70

2.1 CIRCUITO DE COLETORES CONCENTRADORES SOLARES 70

PARABÓLICOS

2.1.1 Processos no circuito de coletores concentradores solares 71 parabólicos

2.1.2 Condições de análise do circuito de coletores concentradores 72 solares parabólicos

2.1.3 Cálculo dos ângulos solares 
2.1.5 Balanço de energia na cobertura 76

2.1.5.1 Condução térmica na cobertura 78

2.1.5.2 Determinação do coeficiente de transferência de calor por 79 convecção entre o ar ambiental e a cobertura, $\mathrm{h}_{\mathrm{c}_{\mathrm{a}}}$

2.1.6 Balanço de energia no tubo absorvedor 81

2.1.6.1 Determinação do coeficiente de transferência de calor por 83 convecção entre o tubo absorvedor e o fluido térmico (HTF), $\mathrm{h}_{\mathrm{c}_{\mathrm{h}}}$

2.1.7 Influência das propriedades radiativas dos materiais 84

2.1.8 Considerações geométricas $\quad 85$

2.1.9 Análise alternativa por resistências térmicas 86

2.1.10 Queda de pressão no fluxo do fluido térmico (HTF) 87

2.2 CIRCUITO DO CICLO RANKINE ORGÂNICO 88

2.2.1 Definição dos processos e estados termodinâmicos do ciclo 88

2.2.2 Parámetros do ciclo 91

2.2.3 Relações de pressões 92

2.2.4 Bomba 92

2.2.5 Expansor 93

2.2.6 Recuperador 93

2.2.6.1 Balanço energético do recuperador 93

2.2.6.2 Características geométricas do recuperador 94

2.2.6.3 Análise do fluxo na tubulação do recuperador 97

2.2.6.4 Análise do fluxo pelas placas do recuperador 99

2.2.6.5 Área de troca de calor no recuperador 100

$\begin{array}{ll}2.2 .7 \text { Condensador } & 101\end{array}$

2.2.7.1 Balanço energético do condensador 102

2.2.7.2 Características geométricas do condensador 103

2.2.7.3 Análise do fluxo na tubulação do condensador 105

2.2.7.4 Análise do fluxo pelas placas na zona superaquecida do 106 condensador

2.2.7.5 Área de troca de calor na zona superaquecida do condensador $\quad 108$ 
2.2.7.6 Análise do fluxo pelas placas na zona saturada do condensador

2.2.7.7 Área de troca de calor na zona saturada do condensador $\quad 110$

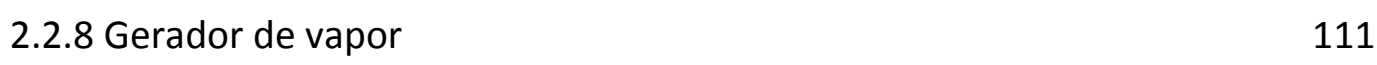

2.2.8.1 Balanço energético do gerador de vapor 112

2.2.8.2 Características geométricas do gerador de vapor 113

2.2.8.3 Análise do fluxo na tubulação do gerador de vapor 115

2.2.8.4 Análise do fluxo pelo casco do gerador de vapor na zona 116 sub-resfriada

2.2.8.5 Área de troca de calor na zona sub-resfriada do gerador de vapor

2.2.8.6 Análise do fluxo pelo casco do gerador de vapor na zona saturada

2.2.8.7 Área de troca de calor na zona saturada do gerador de vapor $\quad 120$

2.2.8.8 Análise do fluxo pelo casco do gerador de vapor na zona superaquecida

2.2.8.9 Área de troca de calor na zona superaquecida do gerador de vapor

2.3 ARMAZENAMENTO TÉRMICO NO CICLO RANKINE ORGÂNICO COM ENERGIA SOLAR

2.3.1 Funcionamento sem utilização de armazenamento térmico

2.3.2 Funcionamento em carga térmica (horas de claridade) 125

2.3.3 Funcionamento em descarga térmica (horas sem luz solar) 126

3 ANÁLISE DOS PARÂMETROS DO MODELO MATEMÁTICO

3.1 CIRCUITO DOS COLETORES CONCENTRADORES PARABÓLICOS 128

3.1.1 Análise do fluido térmico (HTF) no circuito dos coletores solares 128

3.1.1.1 Propriedades termodinâmicas dos fluidos térmicos (HTFs) 129

$\begin{array}{ll}\text { 3.1.2 Radiação solar disponível } & 131\end{array}$

3.1.2.1 Primeira aproximação: radiação extraterrestre 131

3.1.2.2 Ângulos de incidência na horizontal (Zenith) e no plano do 135 coletor 
3.1.2.3 Radiação real 137

3.1.3 Principais medidas geométricas dos coletores concentradores $\quad 138$ parabólicos comerciais

3.1.4 Propriedades do coletor concentrador parabólico 141

3.1.4.1 Fator de intercepção 141

3.1.4.2 Refletância especular 142

3.1.4.3 Transmissividade da cobertura 143

3.1.4.4 Absortância do tubo absorvedor $\quad 144$

3.1.4.5 Emissividade da superfície do tubo absorvedor 145

3.1.4.6 Condutividade térmica do receptor 146

$\begin{array}{ll}\text { 3.1.4.7 Emissividade da cobertura } & 147\end{array}$

$\begin{array}{ll}\text { 3.1.4.8 Condutividade da cobertura } & 147\end{array}$

3.1.4.9 Influência do coeficiente de transferência de calor por 147 convecção entre o ar ambiental e a cobertura, $\mathrm{h}_{\mathrm{c}_{\mathrm{a}}}$

3.1.4.10 Influência do coeficiente de transferência de calor por 148 convecção entre o tubo absorvedor e o $\mathrm{HTF}, \mathrm{h}_{\mathrm{ch}_{\mathrm{h}}}$

3.1.5 Estudo de número de receptores necessários em função da 149 radiação solar incidente e vazão mássica do HTF

3.2 CICLO RANKINE ORGÂNICO 152

3.2.1 Seleção do fluido orgânico do ciclo Rankine orgânico (ORC) 152

3.2.2 Trocadores de calor de tubos e placas 157

3.2.3 Dimensionamento do recuperador 158

3.2.3.1 Para 40\% de eficiência isentrópica do expansor 160

3.2.3.2 Para 50\% de eficiência isentrópica do expansor 161

3.2.3.3 Para 60\% de eficiência isentrópica do expansor 162

3.2.3.4 Para 70\% de eficiência isentrópica do expansor 163

3.2.3.5 Para 80\% de eficiência isentrópica do expansor 164

3.2.3.6 Comentários sobre o dimensionamento do recuperador 165

3.2.4 Dimensionamento do condensador 167

3.2.4.1 Para 40\% de eficiência isentrópica do expansor 167

3.2.4.2 Para 50\% de eficiência isentrópica do expansor 169 
3.2.4.3 Para 60\% de eficiência isentrópica do expansor 170

$\begin{array}{ll}\text { 3.2.4.4 Para 70\% de eficiência isentrópica do expansor } & 171\end{array}$

3.2.4.5 Para 80\% de eficiência isentrópica do expansor 173

3.2.4.6 Para 100\% de eficiência isentrópica do expansor 174

3.2.4.7 Comentários sobre o dimensionamento do condensador 175

$\begin{array}{ll}\text { 3.2.5 Trocador de calor de casco e tubos } & 177\end{array}$

$\begin{array}{ll}\text { 3.2.6 Dimensionamento do gerador de vapor } & 178\end{array}$

3.2.6.1 Para 40\% de eficiência isentrópica do expansor 179

3.2.6.2 Para 50\% de eficiência isentrópica do expansor 181

3.2.6.3 Para 60\% de eficiência isentrópica do expansor 182

3.2.6.4 Para 70\% de eficiência isentrópica do expansor 183

3.2.6.5 Para 80\% de eficiência isentrópica do expansor 185

3.2.6.6 Para 100\% de eficiência isentrópica do expansor 186

3.2.6.7 Comentários sobre o dimensionamento do gerador de vapor $\quad 187$

$\begin{array}{ll}\text { 3.3 ARMAZENAMENTO TÉRMICO } & 189\end{array}$

4 RESULTADOS 191

4.1 CIRCUITO DO CICLO RANKINE ORGÂNICO 191

$\begin{array}{ll}\text { 4.1.1 Recuperador } & 191\end{array}$

4.1.2 Condensador 192

4.1.3 Gerador de vapor 193

4.1.4 Resumo dos parâmetros do ciclo termodinâmico 194

4.2 CIRCUITO DE COLETORES CONCENTRADORES SOLARES 197

PARABÓLICOS

4.2.1 Análise de número de receptores necessários 197

4.3 ARMAZENAMENTO TÉRMICO 200

4.3.1 Possíveis dimensões do sistema de armazenamento térmico 201

4.4 COMPORTAMENTO DA PLANTA 203

4.5 SIMULAÇÃO DE FUNCIONAMENTO COM DADOS REAIS 205

4.5.11 de janeiro 206

$\begin{array}{ll}4.5 .23 \text { de janeiro } & 207\end{array}$ 
5 CONCLUSÕES E SUGESTÕES PARA TRABALHOS FUTUROS

5.1 CONCLUSÕES

211

5.2 SUGESTÕES PARA TRABALHOS FUTUROS

212

6 REFERENCIAS BIBLIOGRÁFICAS 


\section{Lista de figuras}

$\begin{array}{ll}\text { Figura } 1.1 \text { - Espectro solar } & 37\end{array}$

Figura 1.2 - Tipos de radiação incidente 38

Figura 1.3 - Ângulos solares 39

Figura 1.4 - Partes de um coletor concentrador linear parabólico 41

Figura 1.5 - Parábola $\quad 42$

Figura 1.6 - Parábola com vértice num sistema de coordenadas 43 cartesianas

Figura 1.7 - Medidas de um coletor concentrador linear parabólico 44

Figura 1.8 - Reflexão dos raios solares no concentrador parabólico 45

Figura 1.9 Duas parábolas possíveis para uma mesma abertura e igual $\quad 47$ razão geométrica de concentração

Figura 1.10 - Reflexão de luz numa superfície especular perfeita 49

Figura 1.11 - Aproveitamento de vapor superaquecido com 52 recuperador

Figura 1.12 - Esquema do ciclo Rankine orgânico

Figura 1.13 - Diagrama T-s para água gerado em EES 56

Figura 1.14 - Diagramas T-s para os refrigerantes R123 e R124 gerados 56 em EES

Figura 1.15 - ODP para diversos refrigerantes $\quad 58$

Figura 1.16 - Esquema de recuperação de calor implementado pela GE 61

Figura 1.17 - Reservatório geotérmico $\quad 61$

Figura 1.18 - Geração de energia com biomassa 62

Figura 1.19 - Ciclo Rankine orgânico solar com entrada direta de calor 63

Figura 1.20 - Ciclo Rankine orgânico solar com trocador de calor 63

Figura 1.21 - Usina de geração solar direta Rankine 64

Figura 1.22 - Ciclo Rankine orgânico solar comercial de baixa potência 65

Figura 1.23 - Armazenamento direto com dois tanques 66

Figura 1.24 - Armazenamento indireto com dois tanques 67 
Figura 1.25 - Tanque de armazenamento direto com efeito termoclina

Figura 2.1 - Ciclo Rankine orgânico com coletores concentradores solares $\quad 70$

Figura 2.2 - Circuito de coletores concentradores solares parabólicos 71

Figura 2.3 - Cobertura $\quad 77$

Figura 2.4 - Balanço de energia no setor interno da cobertura 79

Figura 2.5 - Tubo absorvedor $\quad 81$

Figura 2.6 - Resistências térmicas $\quad 87$

Figura 2.7 - Circuito do ciclo Rankine orgânico 89

Figura 2.8 - Diagrama T-s do ciclo Rankine orgânico 91

Figura 2.9 - Esquema do recuperador 93

Figura 2.10 - Trocador de tubo e placas 94

Figura 2.11 - Medidas de um trocador de tubo e placas 95

Figura 2.12 - Relação entre aleta quadrada e aleta redonda 97

Figura 2.13 - Esquema do condensador 102

Figura 2.14 - Esquema da zona superaquecida do condensador 102

Figura 2.15 - Esquema da zona saturada do condensador 103

Figura 2.16 - Esquema do gerador de vapor 111

Figura 2.17 - Esquema da zona sub-resfriada do gerador de vapor 112

Figura 2.18 - Esquema da zona saturada do gerador de vapor 113

Figura 2.19 - Esquema da zona superaquecida do gerador de vapor 113

Figura 2.20 - Medidas do trocador de calor de casco e tubos 114

Figura 2.21 - Ciclo orgânico Rankine com armazenamento térmico 126 carregando

Figura 2.22 - Ciclo orgânico Rankine com armazenamento térmico 127 descarregando

Figura 3.1 - Regressão da viscosidade dinâmica a partir de dados de [24] 129

Figura 3.2 - In da viscosidade dinâmica VS. In da temperatura a partir 129 de dados de [24] 
Figura 3.3 - Regressão do calor específico a pressão constante a partir de dados de [24]

Figura 3.4 - Regressão da massa específica a partir de dados de [24] 129

Figura 3.5 - Regressão do condutividade térmica a partir de dados de [24] 130

Figura 3.6 - Ângulo de declinação em função do dia do ano (n) 132

Figura 3.7 - Radiação extraterrestre em função do dia para o meio-dia, 132 latitude $23^{\circ} \mathrm{N}$

Figura 3.8 - Radiação extraterrestre em função do dia para o meio-dia, latitude $23^{\circ} \mathrm{S}$

Figura 3.9 - Radiação extraterrestre em função do dia para o meio-dia, 133 latitude $0^{\circ}$

Figura 3.10 - Radiação extraterrestre em função da hora para o dia 1 do ano, latitude $23^{\circ} \mathrm{N}$

Figura 3.11 - Radiação extraterrestre em função da hora para o dia 1 do ano, latitude $23^{\circ} \mathrm{S}$

Figura 3.12 - Radiação extraterrestre em função da hora para o dia 1 do ano, latitude $0^{\circ}$

Figura 3.13 - Energia radiativa extraterrestre incidente em função do dia entre 11.30 e $12.30 \mathrm{~h}$, latitude $23^{\circ} \mathrm{N}$

Figura 3.14 - Energia radiativa extraterrestre incidente em função do dia entre 11.30 e $12.30 \mathrm{~h}$, latitude $23^{\circ} \mathrm{S}$

Figura 3.15 - Energia radiativa extraterrestre incidente em função do dia entre 11.30 e $12.30 \mathrm{~h}$, latitude $0^{\circ}$

Figura 3.16 - Ângulo $\theta_{z}$ em função do dia para o meio-dia, latitude $23^{\circ} \mathrm{N}$ 135

Figura 3.17 - Ângulo $\theta_{z}$ em função do dia para o meio-dia, latitude $23^{\circ} \mathrm{S} \quad 135$

Figura 3.18 - Ângulo $\theta_{z}$ em função do dia para o meio-dia, latitude $0^{\circ} \quad 136$

Figura 3.19 - R em função da hora para o dia 1 do ano, latitude $23^{\circ} \mathrm{N} \quad 136$

Figura 3.20 - R em função da hora para o dia 1 do ano, latitude $23^{\circ} \mathrm{S} \quad 136$

Figura 3.21 - R em função da hora para o dia 1 do ano, latitude $0^{\circ} \quad 137$

Figura 3.22 - Radiação direta horizontal em função da hora para o dia 1138 do ano, latitude $23^{\circ} \mathrm{S}$ 
Figura 3.23 - Radiação direta no concentrador em função da hora para o dia 1 do ano, latitude $23^{\circ} \mathrm{S}$

Figura 3.24 - Radiação direta horizontal em função da hora para o dia 170 do ano, latitude $23^{\circ} \mathrm{S}$

Figura 3.25 - Radiação direta no concentrador em função da hora para o dia 170 do ano, latitude $23^{\circ} \mathrm{S}$

Figura 3.26 - Características dos coletores Luz

Figura 3.27 - Fator de intercepção vs. razão de concentração

Figura 3.28 - Fator de intercepção vs. desvio padrão

Figura 3.29 - Refletividade dos concentradores Guardian

Figura 3.30 - Refletividade dos concentradores FLABEG 143

Figura 3.31 - Transmissividade do vidro pyrex 144

Figura 3.32 - Tubo absorvedor Siemens UVAC 2010 145

Figura 3.33 - Equações de emissividade para diferentes recobrimentos 146

Figura 3.34 - Emissividade superficial VS. Temperatura para diferentes 146 recobrimentos

Figura 3.35 - Coeficiente de transferência de calor por convecção 148 entre o ar ambiental e a cobertura em função da temperatura de película e a velocidade do vento

Figura 3.36 - Coeficiente de transferência de calor por convecção entre 148 o fluido térmico e a tubo absorvedor em função da vazão mássica XCELTHERM ${ }^{\circledR}$ MK1

Figura 3.37 - Coeficiente de transferência de calor por convecção entre 148 o fluido térmico e a tubo absorvedor em função da vazão mássica SYLTHERM 800

Figura 3.38 - Coeficiente de transferência de calor por convecção entre 149 o fluido térmico e a tubo absorvedor em função da vazão mássica DOWTHERM A

Figura 3.39 - Coeficiente de transferência de calor por convecção entre o fluido térmico e a tubo absorvedor em função da vazão mássica Therminol VP-1 
Figura 3.40 - Eficiência do ciclo orgânico Rankine em função da temperatura máxima, $\eta_{\mathrm{st}}=40 \%$ e $\mathrm{P}_{\mathrm{alta}}=3600 \mathrm{kPa}$

Figura 3.41 - Eficiência do ciclo orgânico Rankine em função da temperatura máxima, $\eta_{\mathrm{st}}=50 \%$ e $\mathrm{P}_{\text {alta }}=3600 \mathrm{kPa}$

Figura 3.42 - Eficiência do ciclo orgânico Rankine em função da temperatura máxima, $\eta_{\mathrm{st}}=60 \%$ e $\mathrm{P}_{\mathrm{alta}}=3600 \mathrm{kPa}$

Figura 3.43 - Eficiência do ciclo orgânico Rankine em função da 155 temperatura máxima, $\eta_{\mathrm{st}}=70 \%$ e $\mathrm{P}_{\mathrm{alta}}=3600 \mathrm{kPa}$

Figura 3.44 - Eficiência do ciclo orgânico Rankine em função da temperatura máxima, $\eta_{\mathrm{st}}=80 \%$ e $\mathrm{P}_{\mathrm{alta}}=3600 \mathrm{kPa}$

Figura 3.45 - Eficiência do ciclo orgânico Rankine em função da temperatura máxima, $\eta_{\mathrm{st}}=90 \%$ e $\mathrm{P}_{\mathrm{alta}}=3600 \mathrm{kPa}$

Figura 3.46 - Eficiência do ciclo orgânico Rankine em função da 155 temperatura máxima, $\eta_{\mathrm{st}}=100 \%$ e $\mathrm{P}_{\mathrm{alta}}=3600 \mathrm{kPa}$

Figura 3.47 - Eficiência do ciclo orgânico Rankine em função da 156 temperatura máxima, $\eta_{\mathrm{st}}=40 \%$ e $\mathrm{P}_{\mathrm{alta}}=0,9 * \mathrm{P}_{\mathrm{cr}}$

Figura 3.48 - Eficiência do ciclo orgânico Rankine em função da temperatura máxima, $\eta_{s t}=50 \%$ e $P_{\text {alta }}=0,9 * P_{c r}$

Figura 3.49 - Eficiência do ciclo orgânico Rankine em função da 156 temperatura máxima, $\eta_{s t}=60 \%$ e $P_{\text {alta }}=0,9 * P_{c r}$

Figura 3.50 - Eficiência do ciclo orgânico Rankine em função da 156 temperatura máxima, $\eta_{s t}=70 \%$ e $P_{\text {alta }}=0,9 * P_{c r}$

Figura 3.51 - Eficiência do ciclo orgânico Rankine em função da temperatura máxima, $\eta_{\mathrm{st}}=80 \%$ e $\mathrm{P}_{\mathrm{alta}}=0,9 * \mathrm{P}_{\mathrm{cr}}$

Figura 3.52 - Eficiência do ciclo orgânico Rankine em função da temperatura máxima, $\eta_{\mathrm{st}}=90 \%$ e $\mathrm{P}_{\mathrm{alta}}=0,9 * \mathrm{P}_{\mathrm{cr}}$

Figura 3.53 - Eficiência do ciclo orgânico Rankine em função da temperatura máxima, $\eta_{\mathrm{st}}=100 \%$ e $\mathrm{P}_{\mathrm{alta}}=0,9 * \mathrm{P}_{\mathrm{cr}}$

Figura 3.54 - Área de transferência de calor do recuperador $\left(\mathrm{m}^{2}\right) 7.75$ $5 / 8 \mathrm{~T}_{\mathrm{st}}=100 \%$ 
Figura 3.55 - Área de transferência de calor do recuperador $\left(\mathrm{m}^{2}\right) 8.0$ -

$$
3 / 8 \mathrm{~T} \eta_{\mathrm{st}}=100 \%
$$

Figura 3.56 - Comprimento dos tubos do recuperador $(\mathrm{m}) 7.75-5 / 8 \mathrm{~T}$ $\eta_{\mathrm{st}}=100 \%$

Figura 3.57 - Comprimento dos tubos do recuperador $(\mathrm{m})$ 8.0 - 3/8 T $\eta_{s t}=100 \%$

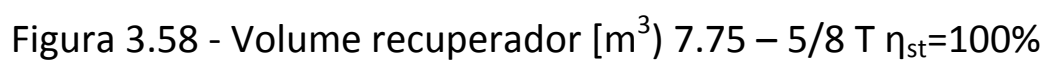

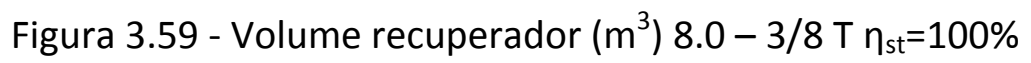

Figura 3.60 - Área de transferência de calor do recuperador $\left(\mathrm{m}^{2}\right) \eta_{\mathrm{st}}=40 \%$

Figura 3.61 - Comprimento dos tubos do recuperador $(m) \eta_{s t}=40 \%$

Figura 3.62 - Volume recuperador $\left(\mathrm{m}^{3}\right) \eta_{\mathrm{st}}=40 \%$

Figura 3.63 - Queda de pressão na tubulação do recuperador $\eta_{s t}=40 \%$

Figura 3.64 - Queda de pressão no casco do recuperador $\eta_{\mathrm{st}}=40 \%$

Figura 3.65 - Área de transferência de calor do recuperador $\left(\mathrm{m}^{2}\right) \eta_{\mathrm{st}}=50 \%$

Figura 3.66 - Comprimento dos tubos do recuperador $(m) \eta_{s t}=50 \%$

Figura 3.67 - Volume recuperador $\left(\mathrm{m}^{3}\right) \eta_{\mathrm{st}}=50 \%$

Figura 3.68 - Queda de pressão na tubulação do recuperador $\eta_{s t}=50 \%$

Figura 3.69 - Queda de pressão no casco do recuperador $\eta_{\mathrm{st}}=50 \%$

Figura 3.70 - Área de transferência de calor do recuperador $\left(\mathrm{m}^{2}\right) \eta_{\mathrm{st}}=60 \%$

Figura 3.71 - Comprimento dos tubos do recuperador $(m) \eta_{s t}=60 \%$

Figura 3.72 - Volume recuperador $\left(\mathrm{m}^{3}\right) \eta_{\mathrm{st}}=60 \%$

Figura 3.73 - Queda de pressão na tubulação do recuperador $\eta_{s t}=60 \%$

Figura 3.74 - Queda de pressão no casco do recuperador $\eta_{\text {st }}=60 \%$

Figura 3.75 - Área de transferência de calor do recuperador $\left(m^{2}\right) \eta_{s t}=70 \%$

Figura 3.76 - Comprimento dos tubos do recuperador $(m) \eta_{s t}=70 \%$ 163

Figura 3.77 - Volume recuperador $\left(\mathrm{m}^{3}\right) \eta_{\mathrm{st}}=70 \%$ 163

Figura 3.78 - Queda de pressão na tubulação do recuperador $\eta_{\mathrm{st}}=70 \%$

Figura 3.79 - Queda de pressão no casco do recuperador $\eta_{\text {st }}=70 \%$ 163

Figura 3.80 - Área de transferência de calor do recuperador $\left(m^{2}\right) \eta_{s t}=80 \%$ 164

Figura 3.81 - Comprimento dos tubos do recuperador $(m) \eta_{s t}=80 \%$ 164

Figura 3.82 - Volume recuperador $\left(\mathrm{m}^{3}\right) \eta_{\mathrm{st}}=80 \%$ 
Figura 3.83 - Queda de pressão na tubulação do recuperador $\eta_{\mathrm{st}}=80 \% \quad 164$

Figura 3.84 - Queda de pressão no casco do recuperador $\eta_{\mathrm{st}}=80 \% \quad 164$

Figura 3.85 - Vazão mássica de água em função da temperatura de 167 saída, $T_{\text {ao }}$

Figura 3.86 - Área de transferência de calor do condensador $\left(m^{2}\right) \eta_{s t}=40 \% \quad 167$

Figura3.87 - Comprimento dos tubos do condensador $(m) \eta_{s t}=40 \% \quad 167$

Figura 3.88 - Volume do condensador $\left(\mathrm{m}^{3}\right) \eta_{\mathrm{st}}=40 \% \quad 168$

Figura 3.89 - Queda de pressão na zona superaquecida do condensador 168

$$
\eta_{s t}=40 \%
$$

Figura 3.90 - Queda de pressão na zona saturada do condensador $\eta_{\mathrm{st}}=40 \% \quad 168$

Figura 3.91 - Queda de pressão na tubulação do condensador $\eta_{\mathrm{st}}=40 \% \quad 168$

Figura 3.92 - Área de transferência de calor do condensador $\left[\mathrm{m}^{2}\right) \eta_{\mathrm{st}}=50 \% \quad 169$

Figura 3.93 - Comprimento dos tubos do condensador $(m) \eta_{s t}=50 \% \quad 169$

Figura 3.94 - Volume do condensador $\left(\mathrm{m}^{3}\right) \eta_{\mathrm{st}}=50 \% \quad 169$

Figura 3.95 - Queda de pressão na zona superaquecida do condensador 169 $\eta_{\mathrm{st}}=50 \%$

Figura 3.96 - Queda de pressão na zona saturada do condensador $\eta_{\mathrm{st}}=50 \% \quad 169$

Figura 3.97 - Queda de pressão na tubulação do condensador $\eta_{\mathrm{st}}=50 \% \quad 170$

Figura 3.98 - Área de transferência de calor do condensador $\left(\mathrm{m}^{2}\right) \eta_{\mathrm{st}}=60 \% \quad 170$

Figura 3.99 - Comprimento dos tubos do condensador $(m) \eta_{s t}=60 \% \quad 170$

Figura 3.100 - Volume do condensador $\left(\mathrm{m}^{3}\right) \eta_{\mathrm{st}}=60 \%$

Figura 3.101 - Queda de pressão na zona superaquecida do condensador 171 $\eta_{s t}=60 \%$

Figura 3.102 - Queda de pressão na zona saturada do condensador $\eta_{\mathrm{st}}=60 \%$

Figura 3.103 - Queda de pressão na tubulação do condensador $\eta_{s t}=60 \%$

Figura 3.104 - Área de transferência de calor do condensador $\left(\mathrm{m}^{2}\right)$ $\eta_{\mathrm{st}}=70 \%$

Figura 3.105 - Comprimento dos tubos do condensador $(m) \eta_{s t}=70 \%$

Figura 3.106 - Volume do condensador $\left(\mathrm{m}^{3}\right) \eta_{\mathrm{st}}=70 \%$ 
Figura 3.107 - Queda de pressão na zona superaquecida do condensador $\eta_{\mathrm{st}}=70 \%$

Figura 3.108- Queda de pressão na zona saturada do condensador $\eta_{\mathrm{st}}=70 \%$

Figura 3.109 - Queda de pressão na tubulação do condensador $\eta_{s t}=70 \%$ 172

Figura 3.110 - Área de transferência de calor do condensador $\left(\mathrm{m}^{2}\right)$ 173 $\eta_{\mathrm{st}}=80 \%$

Figura 3.111 - Comprimento dos tubos do condensador $(m) \eta_{s t}=80 \%$ 173

Figura 3.112 - Volume do condensador $\left(\mathrm{m}^{3}\right) \eta_{\mathrm{st}}=80 \%$ 173

Figura 3.113 - Queda de pressão na zona superaquecida do condensador 173 $\eta_{\mathrm{st}}=80 \%$

Figura 3.114 - Queda de pressão na zona saturada do condensador 173 $\eta_{\mathrm{st}}=80 \%$

Figura 3.115 - Queda de pressão na tubulação do condensador $\eta_{s t}=80 \%$ 174

Figura 3.116 - Área de transferência de calor do condensador $\left(\mathrm{m}^{2}\right)$ 174 $\eta_{\mathrm{st}}=100 \%$

Figura 3.117 - Comprimento dos tubos do condensador $(m) \eta_{s t}=100 \%$ 174

Figura 3.118 - Volume do condensador $\left(\mathrm{m}^{3}\right) \eta_{\mathrm{st}}=100 \%$ 174

Figura 3.119 - Queda de pressão na zona superaquecida do condensador 175 $\eta_{\mathrm{st}}=100 \%$

Figura 3.120 - Queda de pressão na zona saturada do condensador 175 $\eta_{\mathrm{st}}=100 \%$

Figura 3.121 - Queda de pressão na tubulação do condensador $\eta_{\mathrm{st}}=100 \%$ 175

Figura 3.122 - Vazão mássica do fluido térmico em função da 178 temperatura $T_{b} \eta_{s t}=40 \%$

Figura 3.123 - Vazão mássica do fluido térmico em função da temperatura $T_{b} \eta_{s t}=50 \%$

Figura 3.124 - Vazão mássica do fluido térmico em função da temperatura $T_{b} \eta_{s t}=60 \%$

Figura 3.125 - Vazão mássica do fluido térmico em função da temperatura $T_{b} \eta_{s t}=70 \%$ 
Figura 3.126 - Vazão mássica do fluido térmico em função da temperatura $T_{b} \eta_{s t}=80 \%$

Figura 3.127 - Vazão mássica do fluido térmico em função da temperatura $T_{b} \eta_{s t}=100 \%$

Figura 3.128 - Área de transferência de calor do gerador de vapor $\left(\mathrm{m}^{2}\right)$ 179 $\eta_{\text {st }}=40 \%$ (XCELTHERM ${ }^{\circledR}$ MK1, DOWTHERM A e Therminol VP-1)

Figura 3.129 - Área de transferência de calor do gerador de vapor $\left(\mathrm{m}^{2}\right)$ 179 $\eta_{\mathrm{st}}=40 \%($ SYLTHERM 800$)$

Figura 3.130 - Comprimento dos tubos do gerador de vapor $(m) \eta_{s t}=40 \%$ 180 (XCELTHERM ${ }^{\circledR}$ MK1, DOWTHERM A e Therminol VP-1)

Figura 3.131 - Comprimento dos tubos do gerador de vapor $(\mathrm{m})$ 180 $\eta_{\mathrm{st}}=40 \%$ (SYLTHERM 800)

Figura 3.132 - Volume do gerador de vapor $\left(\mathrm{m}^{3}\right) \eta_{\mathrm{st}}=40 \%\left(\right.$ XCELTHERM $^{\circledR}$ 180 MK1, DOWTHERM A e Therminol VP-1)

Figura 3.133 - Volume do gerador de vapor $\left(\mathrm{m}^{3}\right) \eta_{\mathrm{st}}=40 \%$ (SYLTHERM 800) 180 Figura 3.134 - Queda de pressão no casco do gerador de vapor $\eta_{\mathrm{st}}=40 \% \quad 180$

Figura 3.135 - Área de transferência de calor do gerador de vapor $\left(\mathrm{m}^{2}\right)$ 181 $\eta_{s t}=50 \%$ (XCELTHERM ${ }^{\circledR}$ MK1, DOWTHERM A e Therminol VP-1)

Figura 3.136 - Área de transferência de calor do gerador de vapor $\left(\mathrm{m}^{2}\right)$ 181 $\eta_{s t}=50 \%$ (SYLTHERM 800)

Figura 3.137 - Comprimento dos tubos do gerador de vapor $(m) \eta_{s t}=50 \%$ (XCELTHERM ${ }^{\circledR}$ MK1, DOWTHERM A e Therminol VP-1)

Figura 3.138 - Comprimento dos tubos do gerador de vapor $(\mathrm{m})$ 181 $\eta_{\mathrm{st}}=50 \%$ (SYLTHERM 800)

Figura 3.139 - Volume do gerador de vapor $\left(\mathrm{m}^{3}\right) \eta_{\text {st }}=50 \%\left(\right.$ XCELTHERM $^{\circledR}$ 181 MK1, DOWTHERM A e Therminol VP-1)

Figura 3.140 - Volume do gerador de vapor $\left(\mathrm{m}^{3}\right) \eta_{\mathrm{st}}=50 \%$ (SYLTHERM 800) 181

Figura 3.141 - Queda de pressão no casco do gerador de vapor $\eta_{s t}=50 \%$ 
Figura 3.142 - Área de transferência de calor do gerador de vapor $\left(\mathrm{m}^{2}\right)$ $\eta_{\text {st }}=60 \%$ (XCELTHERM ${ }^{\circledR}$ MK1, DOWTHERM A e Therminol VP-1)

Figura 3.143 - Área de transferência de calor do gerador de vapor $\left(\mathrm{m}^{2}\right)$ $\eta_{\mathrm{st}}=60 \%$ (SYLTHERM 800)

Figura 3.144 - Comprimento dos tubos do gerador de vapor $(m) \eta_{s t}=60 \%$ 182 (XCELTHERM ${ }^{\circledR}$ MK1, DOWTHERM A e Therminol VP-1)

Figura 3.145 - Comprimento dos tubos do gerador de vapor $(m) \eta_{s t}=60 \%$ 182 (SYLTHERM 800)

Figura 3.146 - Volume do gerador de vapor $\left(\mathrm{m}^{3}\right) \eta_{\mathrm{st}}=60 \%\left(\right.$ XCELTHERM $^{\circledR}$ 183 MK1, DOWTHERM A e Therminol VP-1)

Figura 3.147 - Volume do gerador de vapor $\left(\mathrm{m}^{3}\right) \eta_{\mathrm{st}}=60 \%$ (SYLTHERM 800) 183

Figura 3.148 - Queda de pressão no casco do gerador de vapor $\eta_{\mathrm{st}}=60 \% \quad 183$

Figura 3.149 - Área de transferência de calor do gerador de vapor $\left(\mathrm{m}^{2}\right)$ 183 $\eta_{\text {st }}=70 \%\left(\right.$ XCELTHERM $^{\circledR}$ MK1, DOWTHERM A e Therminol VP-1)

Figura 3.150 - Área de transferência de calor do gerador de vapor $\left(\mathrm{m}^{2}\right)$ 183 $\eta_{\mathrm{st}}=70 \%$ (SYLTHERM 800)

Figura 3.151 - Comprimento dos tubos do gerador de vapor $(m) \eta_{s t}=70 \%$ 184 (XCELTHERM ${ }^{\circledR}$ MK1, DOWTHERM A e Therminol VP-1)

Figura 3.152 - Comprimento dos tubos do gerador de vapor $(m) \eta_{s t}=70 \%$ 184 (SYLTHERM 800)

Figura 3.153 - Volume do gerador de vapor $\left(\mathrm{m}^{3}\right) \eta_{\mathrm{st}}=70 \%\left(\right.$ XCELTHERM $^{\circledast}$ 184 MK1, DOWTHERM A e Therminol VP-1)

Figura 3.154 - Volume do gerador de vapor $\left(\mathrm{m}^{3}\right) \eta_{\text {st }}=70 \%$ (SYLTHERM 800) 184

Figura 3.155 - Queda de pressão no casco do gerador de vapor $\eta_{\mathrm{st}}=70 \% \quad 184$

Figura 3.156 - Área de transferência de calor do gerador de vapor $\left(\mathrm{m}^{2}\right) \quad 185$ $\eta_{\text {st }}=80 \%\left(\right.$ XCELTHERM $^{\circledR}$ MK1, DOWTHERM A e Therminol VP-1)

Figura 3.157 - Área de transferência de calor do gerador de vapor $\left(\mathrm{m}^{2}\right)$ $\eta_{\mathrm{st}}=80 \%($ SYLTHERM 800) 
Figura 3.158 - Comprimento dos tubos do gerador de vapor $(m) \eta_{s t}=80 \%$

(XCELTHERM ${ }^{\circledast}$ MK1, DOWTHERM A e Therminol VP-1)

Figura 3.159 - Comprimento dos tubos do gerador de vapor $(m) \eta_{s t}=80 \%$ (SYLTHERM 800)

Figura 3.160 - Volume do gerador de vapor $\left(\mathrm{m}^{3}\right) \eta_{\mathrm{st}}=80 \%\left(\right.$ XCELTHERM $^{\circledR}$

MK1, DOWTHERM A e Therminol VP-1)

Figura 3.161 - Volume do gerador de vapor $\left(\mathrm{m}^{3}\right) \eta_{\mathrm{st}}=80 \%$ (SYLTHERM 800) 185

Figura 3.162 - Queda de pressão no casco do gerador de vapor $\eta_{\mathrm{st}}=80 \% \quad 186$

Figura 3.163 - Área de transferência de calor do gerador de vapor $\left(\mathrm{m}^{2}\right) \quad 186$ $\eta_{s t}=100 \%$ (XCELTHERM $^{\circledR}$ MK1, DOWTHERM A e

Therminol VP-1)

Figura 3.164 - Área de transferência de calor do gerador de vapor $\left(\mathrm{m}^{2}\right)$ $\eta_{\text {st }}=100 \%$ (SYLTHERM 800)

Figura 3.165 - Comprimento dos tubos do gerador de vapor $(m) \eta_{s t}=100 \%$ (XCELTHERM ${ }^{\circledR}$ MK1, DOWTHERM A e Therminol VP-1)

Figura 3.166 - Comprimento dos tubos do gerador de vapor $(m) \eta_{s t}=100 \%$ (SYLTHERM 800)

Figura 3.167 - Volume do gerador de vapor $\left(\mathrm{m}^{3}\right) \eta_{\mathrm{st}}=100 \%$

(XCELTHERM ${ }^{\circledR}$ MK1, DOWTHERM A e Therminol VP-1)

Figura 3.168 - Volume do gerador de vapor $\left(\mathrm{m}^{3}\right) \eta_{\mathrm{st}}=100 \%($ SYLTHERM 800)

Figura 3.169 - Queda de pressão no casco do gerador de vapor $\eta_{s t}=100 \%$ 187

Figura 3.170 - Formas de energia numa planta solar com 190 armazenamento e energia fóssil auxiliar

Figura 4.1 - Esquema de troca de calor no recuperador

Figura 4.2 - Fração de superaquecimento aproveitada vs. temperatura de saída do lado quente do recuperador

Figura 4.3 - Esquema de troca de calor no condensador

Figura 4.4 - Esquema de troca de calor no gerador de vapor 193

Figura 4.5 - Diagrama Temperatura (T) vs. Entropia (s) para os ciclos 195 orgânicos Rankine estudados 
Figura 4.6 - Calor rejeitado e potência gerada para um ORC com $\eta_{s t}=40 \%$ como fração da energia fornecida ao ciclo

Figura 4.7 - Calor rejeitado e potência gerada para um ORC com $\eta_{s t}=50 \%$ como fração da energia fornecida ao ciclo

Figura 4.8 - Calor rejeitado e potência gerada para um ORC com $\eta_{s t}=60 \%$ como fração da energia fornecida ao ciclo

Figura 4.9 - Calor rejeitado e potência gerada para um ORC com $\eta_{s t}=70 \%$ como fração da energia fornecida ao ciclo

Figura 4.10 - Calor rejeitado e potência gerada para um ORC com $\eta_{s t}=80 \%$ como fração da energia fornecida ao ciclo

Figura 4.11 - Calor rejeitado e potência gerada para um ORC com $\eta_{s t}=100 \%$ como fração da energia fornecida ao ciclo

Figura 4.12 - Eficiência do ciclo termodinâmico em função da 197 temperatura $T_{6}$ para diferentes eficiências isentrópicas do expansor

Figura 4.13 - Fluxos energéticos de ganho para o $\mathrm{HTF}\left(\dot{\mathrm{Q}}_{\mathrm{c}_{\mathrm{a}-\mathrm{htf}}}\right)$, perdas 199 térmicas $\left(\dot{Q}_{\mathrm{p}}\right)$ e perdas ópticas do coletor, considerando 50 receptores

Figura 4.14 - Eficiência térmica do circuito de coletores $\left(\eta_{c}\right)$ vs. radiação direta no plano de abertura do coletor

Figura 4.15 - Vazão mássica do HTF vs. Radiação direta no plano de 200 abertura

Figura 4.16 - Esquema de troca de calor entre o HTF e o sal fundido em modo de carga

Figura 4.17 - Esquema de troca de calor entre o HTF e o sal fundido em modo de descarga

Figura 4.18 - Radiação total na horizontal para os dias 1, 2, 3, 4 e 5 de janeiro / 2012

Figura 4.19 - Radiações diretas horizontal $\left(G_{b}\right)$ e no concentrador $\left(G_{a}\right)$ calculadas a partir da medição para o dia 1 de janeiro, no Rio de Janeiro (latitude $23^{\circ} \mathrm{S}$ ) 
Figura 4.20 - Radiações diretas horizontal $\left(G_{b}\right)$ e no concentrador $\left(G_{a}\right)$

calculadas a partir da medição para o dia 3 de janeiro, no

Rio de Janeiro (latitude $23^{\circ} \mathrm{S}$ )

Figura 4.21 - Radiações diretas horizontal $\left(G_{b}\right)$ e no concentrador $\left(G_{a}\right)$

calculadas a partir da medição para o dia 4 de janeiro, no Rio de Janeiro (latitude $23^{\circ} \mathrm{S}$ ) 


\section{Lista de tabelas}

Tabela 2.1 - Estados termodinâmicos no circuito de coletores 71

Tabela 2.2 - Constantes para o cálculo de Nusselt para fluxo externo 80

Tabela 2.3 - Estados termodinâmicos do circuito do ciclo Rankine 89 orgânico

Tabela 3.1 - Características dois principais fluidos térmicos (HFs) estudados

Tabela 3.2 - Equações das propriedades termodinâmicas dos HTFs estudados

Tabela 3.3 - Características dos coletores concentradores parabólicos Euro Trough

Tabela 3.4 - Características geométricas de outros coletores concentradores parabólicos

Tabela 3.5 - Principais parâmetros atuais para o desenho de coletores concentradores parabólicos

Tabela 3.6 - Resultados da simulação no circuito de coletores solares para radiação incidente de $1 \mathrm{~kW} / \mathrm{m}^{2}$ e diferentes vazões mássicas

Tabela 3.7 - Resultados da simulação no circuito de coletores solares para radiação incidente de $0,8 \mathrm{~kW} / \mathrm{m}^{2}$ e diferentes vazões mássicas

Tabela 3.8 - Resultados da simulação no circuito de coletores solares para radiação incidente de $0,5 \mathrm{~kW} / \mathrm{m}^{2}$ e diferentes vazões mássicas

Tabela 3.9 - Resultados da simulação no circuito de coletores solares para radiação incidente de $0,3 \mathrm{~kW} / \mathrm{m}^{2}$ e diferentes vazões mássicas

Tabela 3.10 - Características dos principais refrigerantes 
Tabela 3.11 - Refrigerantes escolhidos para determinar seu comportamento no ORC

Tabela 3.12 - Características dos tipos de trocadores de calor de tubos e placas estudados

Tabela 3.13 - Funções do fator j de Colburn e do fator de atrito para os 158 trocadores de calor de tubos e placasa estudados

Tabela 3.14 - Características dos trocadores de calor de casco e um passo em tubos estudados

Tabela 3.15 - Características dos principais sais fundidos utilizados no 189 armazenamento térmico

Tabela 4.1 - Principais parâmetros resultantes para o recuperador 192

Tabela 4.2 - Principais parâmetros resultantes para o condensador 193

Tabela 4.3 - Principais parâmetros resultantes para o gerador de vapor 194

Tabela 4.4 - Principais parâmetros característicos do ciclo termodinâmico

Tabela 4.5 - Calor rejeitado, potência gerada e energia fornecida ao 195 ciclo ORC para diferentes $\eta_{\text {st }}$

Tabela 4.6- Número de receptores necessários para o ciclo de 50 kW de 198 potência para diferentes fluxos de radiação no plano de abertura para os HTFs: XCELTHERM ${ }^{\circledR}$ MK1, DOWTHERM A, Therminol VP-1

Tabela 4.7 - Número de receptores necessários para o ciclo de 50 kW de 198 potência para diferentes fluxos de radiação no plano de abertura para o HTF: SYLTHERM 800

Tabela 4.8 - Resultados para 50 receptores considerando distintos níveis de radiação incidente no plano de abertura do coletor $\left(G_{a}\right)$

Tabela 4.9 - Temperaturas nos processos de carga e descarga no 201 armazenamento térmico

Tabela 4.10 - Vazão mássica do sal fundido necessária para manter a potência nominal 
Tabela 4.11 - Massa de sal fundido necessária para 6 horas de armazenamento térmico

Tabela 4.12 - Massa de sal fundido necessária para 8 horas de armazenamento térmico

Tabela 4.13 - Volume de sal fundido necessário para 6 horas de 203 armazenamento térmico

Tabela 4.14 - Volume de sal fundido necessário para 8 horas de 203 armazenamento térmico

Tabela 4.15 - Análise de vazões mássicas e potências do ciclo 204 considerando dois conjuntos em paralelo de 50 receptores cada

Tabela 4.16 - Diagrama de eficiência total da planta 205

Tabela 4.17 - Radiação direta no plano de aberura do coletor $\left(G_{a}\right)$ e na 207 horizontal $\left(G_{b}\right)$ para o dia 1 de janeiro de 2012

Tabela 4.18 - Radiação direta no plano de abertura do coletor $\left(G_{a}\right)$ e na 208 horizontal $\left(G_{b}\right)$, e vazões mássicas de HTF resultantes para o dia 3 de janeiro de 2012

Tabela 4.19 - Radiação direta no plano de abertura do coletor $\left(G_{a}\right)$ e na horizontal $\left(G_{b}\right)$, e vazões mássicas de HTF resultantes para o dia 4 de janeiro de 2012 


\title{
Símbolos
}

\author{
A: área \\ a: abertura \\ C: razão geométrica de concentração solar \\ $\mathrm{C}_{\mathrm{P}}$ : calor específico a pressão constante \\ D, d: diâmetro \\ Ė: transferência de energia \\ F: fator de correção do trocador de calor \\ f: função de, fator de atrito, distância focal \\ G: irradiância, velocidade de massa \\ g: aceleração da gravidade \\ h: coeficiente de transferência de calor por convecção, entalpia específica \\ I: energia radiativa por unidade de área \\ $\mathrm{j}_{\mathrm{H}}$ : fator $\mathrm{j}$ de Colbert \\ k: condutividade térmica \\ L, I: comprimento \\ m: massa \\ ṁ: vazão massica \\ Nu: número de Nusselt \\ P: pressão, efetividade de temperatura, perímetro \\ Pr: número de Prandtl \\ Q: fluxo de calor \\ q: calor específico \\ R: razão da taxa de capacidade calorífica \\ r: radio \\ Ra: número de Rayleigh \\ Ra*: número de Rayleigh modificado \\ Re: número de Reynolds \\ S: parâmetro para cálculo de fator de correção do trocador
}


s: entropia específica

St: número de Stanton

$\mathrm{T}$ : temperatura

t: tempo

U: coeficiente global de transferência de calor

V: velocidade

$\overline{\mathrm{V}}$ : volume

v: volume específico

W: parâmetro para cálculo de fator de correção do trocador

$\dot{\mathrm{W}}$ : taxa de trabalho

w: trabalho específico

x: título 


\section{Letras gregas}

$\alpha$ : difusividade térmica, absortância

$\beta$ : coeficiente de expansão volumétrica, ângulo de inclinação do coletor

Y: ângulo de azimute, fator de intercepção

$\Delta$ : variação

ठ: ângulo de declinação

$\varepsilon$ : emissividade, efetividade

$\eta$ : eficiencia

$\theta$ : ângulo de incidência

$\mu$ : Viscosidade dinâmica

v: Viscosidade cinemática

$\rho:$ Massa específica, refletância especular

$\sigma:$ constante de Steffan-Boltzmann

$\varnothing$ : ângulo de latitude

$\tau$ : transmissividade

$\omega$ : ângulo horário 


\section{Subscritos}

0 : inicial

a: absorvedor

b: direta na horizontal, suporte do elemento coletor, bomba

c: convecção, condensador

col: coletor

cr: referente ao ponto crítico

D: referente ao diâmetro

d: modo descarga

e: exterior, equivalente

ef: efetiva

$f:$ final

g: gerador de vapor

htf: fluido térmico

i: interior

k: condução

L: referente ao comprimento

liq: líquido

o: saída

opt: óptica

r: radiação, regenerador

s: solar, isentrópico, modo carga

sky: céu

t: expansor

v: cobertura

$\infty$ : ar ambiental 